\title{
The study of three dimensional radiative mhd casson nanofluid over an exponential porous stretching sheet with heat source under convective boundary conditions
}

\author{
Prathi V. Kumar ${ }^{1}$, Shaik M. Ibrahim ${ }^{*}$, Giulio Lorenzini ${ }^{2}$ \\ ${ }^{1}$ Department of Mathematics, GITAM Deemed to be University, Visakhapatnam, Andhra Pradesh 530045, India \\ ${ }^{2}$ Department of Engineering and Architecture, University of Parma, Parco Area delle Scienze 181/A Parma 43124, Italy
}

Corresponding Author Email: ibrahimsvu@gmail.com

https://doi.org/10.18280/ijht.360101

Received: 10 December 2017

Accepted: 7 March 2018

\section{Keywords:}

three dimensional flow, casson fluid, exponentially stretching sheet, radiation, HAM.

\begin{abstract}
The aspire of this study is to analyse the magnetohydrodynamic (MHD) three dimensional flow of nanofluid induced by an exponentially stretching sheet in the presence of radiation and heat source. Casson fluid model is espoused in this discussion. The repercussion of porous matrix on the Casson nanofluid is also considered. Analysis is carried out when the surface shows convective condition. Brownian motion and thermophoresis effects are considered. Governing equations are evolved and converted into ordinary differential equations using similarity transformations. We adopted homotopy analysis method (HAM) to pick up the solutions. Impact of various parameters is displayed through graphs and tables and discussed in detail. Dual solutions are established by taking Casson and Newtonian fluids. A comparison is made and the correspondence between the acquired and previous results reveals that they are in good correlation.
\end{abstract}

\section{INTRODUCTION}

The low thermal conductivity of conventional heat transfer fluids like water, engine oil, glycol, etc, made them incompetent to attain the modern cooling requirements. Nanofluids (homogeneous mixture of base fluid and nanoparticles) which carry high thermal conductivity are contributed as nonpareil coolants in various fields such as nuclear reactors, heat exchangers, micro-channel heat sinks, polymer extrusion, etc. The term 'nanofluid' was first described by Choi [1]. Prabhat et al. [2] studied the aberrant increase of thermal conductivity and viscosity in nanofluids. Khan and Pop [3] led to the study of nanofluid flow over a stretching sheet. Hady et al. [4] reported the radiation effect on nanofluid flow over a nonlinear stretching sheet. Rida et al. [5] made an analytical study on MHD nanofluid over a stretching sheet through a porous medium under thermal radiation and heat generation. Some recent studies concerning the flow of nanofluid fluid can be found in [6-9].

Three dimensional flow of non-Newtonian fluid plays a vital role in the fields like food processing, performance of lubricants, polymer processing, etc. Wang [10] induced the problem of three dimensional flow over a stretching surface. Ariel [11] obtained an approximate solution to the three dimensional flow past a stretching sheet. Liu et al. [12] analysed the boundary layer flow and heat transfer behaviour of three dimensional fluid flow over an exponentially stretching sheet. The above discussion can be found in Hayat et al. [13], Nadeem et al. [14], Jayachandra babu and Sandeep [15], Ahmed et al. [16] and soon.

Many researchers have allured the concept of Casson fluid [17] as they have gigantic applications in the fields like polymer processing, biomechanics, drilling operations, metallurgy, etc. Casson fluid constitutive equation delineate a nonlinear relationship among stress and rate of strain and has been observed to be perfectly admissible to silicon suspensions, suspensions of bentonite in water, and lithographic varnishes used for printing inks. Mukhopadhyay [18] reported that the Casson parameter depreciates the velocity field. Mustafa and Khan [19] discussed the magnetic field effect on Casson nanofluid over a nonlinearly stretching sheet. Ibrahim and Makinde [20] discussed the stagnation point flow of Casson nanofluid subject to slip and convective boundary conditions. Extensive literature on nanofluid flows can be found in [21-25].

Here we made an investigation to study the MHD three dimensional flow of Casson nanofluid over an exponentially stretching sheet. In the mathematical model convective condition, radiation and heat source effects are also considered. The obtained model was solved by HAM. It has been evinced that this technique is a magnificent approach in dealing with various problems [26-30].

\section{MATHEMATICAL FORMULATION}

Three dimensional steady incompressible MHD flow of Casson nanofluid in a porous medium over an exponentially stretching sheet with convective boundary condition under radiation and heat source is considered. The fluid is electrically conducting under the impact of uniform magnetic field $B_{o}$ which is applied along the Z-direction. Induced magnetic field is not taken into account due to small Reynolds number. Thermophoresis and Brownian motion effects are considered. Physical model of the flow problem is given in Figure 1. 


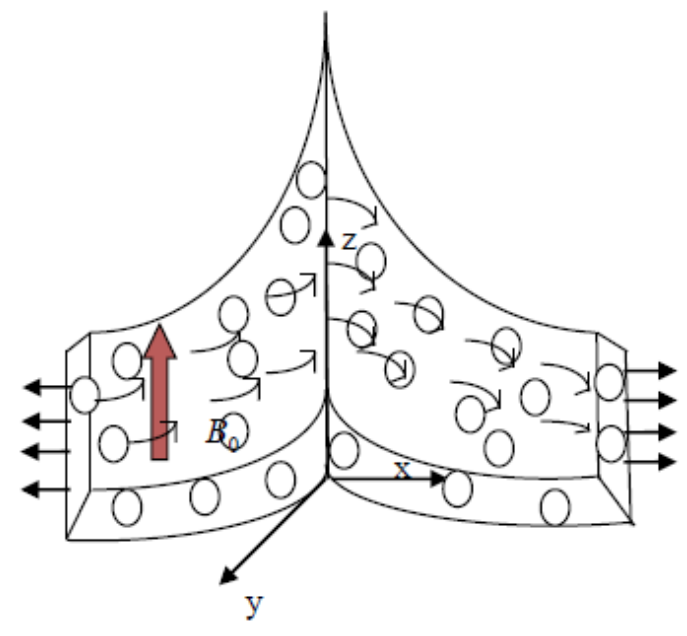

Figure 1. Physical model

The rheological equation of state for an isotropic and incompressible flow of Casson fluid is

$$
\tau_{i j}=\left[\begin{array}{ll}
2\left(\mu_{B}+\frac{p_{y}}{\sqrt{2 \pi}}\right) e_{i j}, & \pi>\pi_{c} \\
2\left(\mu_{B}+\frac{p_{y}}{\sqrt{2 \pi_{c}}}\right) e_{i j}, & \pi_{c}>\pi
\end{array}\right.
$$

where $\mu_{B}$ is plastic dynamic viscosity of the non-Newtonian fluid, $p_{y}$ is the yield stress of the fluid, $\pi$ is the product of the component of deformation rate with itself, $\pi=e_{i j} e_{i j}, e_{i j}$ is the $(i, j)^{\text {th }}$ component of the deformation rate and $\pi_{c}$ is a critical value of this product, based on the non-Newtonian model. Under these assumptions, the equations governing the flow can be written as

$$
\frac{\partial u}{\partial x}+\frac{\partial v}{\partial y}+\frac{\partial w}{\partial z}=0
$$

$u \frac{\partial u}{\partial x}+v \frac{\partial u}{\partial y}+w \frac{\partial u}{\partial z}=v_{f}\left(1+\frac{1}{\beta}\right) \frac{\partial^{2} u}{\partial z^{2}}-\frac{\sigma B_{0}^{2} u}{\rho_{f}}-\frac{v_{f} u}{K^{*}}$

$u \frac{\partial u}{\partial x}+v \frac{\partial u}{\partial y}+w \frac{\partial u}{\partial z}=v_{f}\left(1+\frac{1}{\beta}\right) \frac{\partial^{2} v}{\partial z^{2}}-\frac{\sigma B_{0}^{2} v}{\rho_{f}}-\frac{v_{f} v}{K^{*}}$

$u \frac{\partial T}{\partial x}+v \frac{\partial T}{\partial y}+w \frac{\partial T}{\partial z}=\alpha_{f} \frac{\partial^{2} T}{\partial z^{2}}$

$+\tau\left[D_{B} \frac{\partial C}{\partial z} \frac{\partial T}{\partial z}+\frac{D_{T}}{T_{\infty}}\left(\frac{\partial T}{\partial z}\right)^{2}\right]-\frac{1}{(\rho c)_{f}}\left(\frac{\partial q_{r}}{\partial z}\right)$,

$$
+\frac{Q_{0}}{(\rho c)_{f}}\left(T-T_{\infty}\right)
$$

$u \frac{\partial C}{\partial x}+v \frac{\partial C}{\partial y}+w \frac{\partial C}{\partial z}=D_{B} \frac{\partial^{2} C}{\partial z^{2}}+\frac{D_{T}}{T_{\infty}} \frac{\partial^{2} T}{\partial z^{2}}$

The boundary conditions are

$$
\begin{aligned}
& u=U_{w}=U_{0} e^{\frac{x+y}{L}}, v=V_{w}=V_{0} e^{\frac{x+y}{L}}, w=0, \\
& -k_{f}\left(\frac{\partial T}{\partial z}\right)=h_{f}\left(T_{f}-T\right), C=C_{w} \text { at } z=0, \\
& u \rightarrow 0, v \rightarrow 0 \quad T \rightarrow T_{\infty}, \quad C \rightarrow C_{\infty} \text { as } z \rightarrow \infty .
\end{aligned}
$$

where $\alpha_{f}=\frac{k_{f}}{(\rho c)_{f}}, \tau=\frac{(\rho c)_{p}}{(\rho c)_{f}}$.

Using Rosseland approximation,

$q_{r}=-\frac{4 \sigma^{*}}{3 k^{*}} \frac{\partial T^{4}}{\partial y}=\frac{16 \sigma^{*} T_{\infty}^{3}}{3(\rho c)_{f} k^{*}} \frac{\partial^{2} T}{\partial z^{2}}$

Now we induce the similarity transformations

$$
\begin{aligned}
& u=U_{0} e^{\frac{x+y}{L}} f^{\prime}(\xi), v=U_{0} e^{\frac{x+y}{L}} g^{\prime}(\xi), \\
& w=-\sqrt{\frac{v_{f} U_{0}}{2 L}} e^{\frac{x+y}{2 L}}\left[f(\xi)+\xi f^{\prime}(\xi)+g(\xi)+\xi g^{\prime}(\xi)\right] \\
& \xi=z \sqrt{\frac{U_{0}}{2 v_{f} L}} e^{\frac{x+y}{2 L}}, \theta(\xi)=\frac{T-T_{\infty}}{T_{f}-T_{\infty}}, \varphi(\xi)=\frac{C-C_{\infty}}{C_{w}-C_{\infty}},
\end{aligned}
$$

Here $u, v$ and $w$ satisfy the continuity equation. Eqs. (2) to (6) become

$$
\left(1+\frac{1}{\beta}\right) f^{\prime \prime \prime}+(f+g) f^{\prime \prime}-2\left(f^{\prime}+g^{\prime}\right) f^{\prime}-(M+K) f^{\prime}=0,
$$

$\left(1+\frac{1}{\beta}\right) g^{\prime \prime}+(f+g) g^{\prime \prime}-2\left(f^{\prime}+g^{\prime}\right) g^{\prime}-(M+K) g^{\prime}=0$,

$\left(1+\frac{4 R}{3}\right) \theta^{\prime \prime}+\operatorname{Pr}\left[(f+g) \theta^{\prime}+N b \theta^{\prime} \varphi^{\prime}+N t \theta^{\prime 2}+Q \theta\right]=0$,

$\varphi^{\prime \prime}+S c(f+g) \varphi^{\prime}+\frac{N t}{N b} \theta^{\prime \prime \prime}=0$.

The boundary conditions are

$$
\begin{aligned}
& f(0)=0, g(0)=0, f^{\prime}(0)=1, g^{\prime}(0)=\delta, \\
& \theta^{\prime}(0)=-B_{i}(1-\theta(0)), \varphi(0)=1, \\
& f^{\prime}(\infty) \rightarrow 0, g^{\prime}(\infty) \rightarrow 0, \theta(\infty) \rightarrow 0, \varphi(\infty) \rightarrow 0 .
\end{aligned}
$$

where $M=\frac{2 \sigma B_{0}^{2} L}{\rho_{f} U_{w}}, K=\frac{2 v_{f} L}{U_{w} K^{*}}, \delta=\frac{V_{0}}{U_{0}}, R=\frac{4 \sigma^{*} T_{\infty}^{3}}{k^{*} k}$,

$\operatorname{Pr}=\frac{v_{f}}{\alpha_{f}} \quad, \quad N b=\frac{\tau D_{B}\left(C_{w}-C_{\infty}\right)}{v_{f}} \quad, \quad N t=\frac{\tau D_{B}\left(T_{f}-T_{\infty}\right)}{T_{\infty} v_{f}}$, $Q=\frac{2 Q_{0} L}{U_{w}(\rho c)_{f}}, B_{i}=\frac{h_{f}}{k_{f}} \sqrt{\frac{2 v_{f} L}{U_{w}}}, S c=\frac{v_{f}}{D_{B}}$. 
Non-dimensional skin friction coefficient in $x$ and $y$ directions $C_{f x}$ and $C_{f y}$, local Nusselt number $N u_{x}$ and local Sherwood number $S h_{x}$ are

$$
C_{f x}=\frac{2 \tau_{w x}}{\rho_{f} U_{w}^{2}}, C_{f y}=\frac{2 \tau_{w y}}{\rho_{f} U_{w}^{2}}
$$

where $\tau_{w y}=\mu_{f}\left(1+\frac{1}{\beta}\right)\left(\frac{\partial u}{\partial z}+\frac{\partial w}{\partial x}\right)_{z=0}$,

$\tau_{w y}=\mu_{f}\left(1+\frac{1}{\beta}\right)\left(\frac{\partial v}{\partial z}+\frac{\partial w}{\partial y}\right)_{z=0}, N u_{x}=\frac{x q_{w}}{k_{f}\left(T_{f}-T_{\infty}\right)}$,

$q_{w}=-\left(k_{f}+\left(\frac{16 \sigma^{*} T_{\infty}^{3}}{3 k^{*}}\right)\left(\frac{\partial T}{\partial z}\right)\right)_{z=0}$ and $S h_{x}=\frac{x q_{m}}{D_{B}\left(C_{w}-C_{\infty}\right)}$,

$q_{m}=-D_{B}\left(\frac{\partial C}{\partial z}\right)_{z=0}$.

Substituting $q_{w}$ and $q_{m}$ in the preceding equations, we get

$\left(\frac{\mathrm{Re}}{2}\right)^{1 / 2} C_{f x}=\left(1+\frac{1}{\beta}\right) f^{\prime \prime}(0),\left(\frac{\mathrm{Re}}{2}\right)^{1 / 2} C_{f y}=\left(1+\frac{1}{\beta}\right) g^{\prime \prime}(0)$

$\left(\frac{\mathrm{Re}}{2}\right)^{-1 / 2} \frac{L}{x} N u_{x}=-\left(1+\frac{4}{3} R\right) \theta^{\prime}(0)$

and $\left(\frac{\mathrm{Re}}{2}\right)^{-1 / 2} \frac{L}{x} S h_{x}=-\varphi^{\prime}(0)$

where $\operatorname{Re}=\frac{U_{w} L}{v_{f}}$ is the local Reynolds number.

\section{HAM}

To capture the homotopic solutions of Eqs. (8) to (12), we pick up the initial guesses and linear operators as follows:

$$
\begin{aligned}
& f_{0}(\xi)=\left(1-e^{-\xi}\right), \\
& g_{0}(\xi)=\delta\left(1-e^{-\xi}\right) \\
& \theta_{0}(\xi)=\left(\frac{B i e^{-\xi}}{1+B i}\right), \\
& \varphi_{0}(\xi)=e^{-\xi} . \\
& L_{1}(f)=f^{\prime \prime}-f^{\prime}, \\
& L_{2}(f)=g^{\prime \prime \prime}-g^{\prime} \\
& L_{3}(\theta)=\theta^{\prime \prime}-\theta, \\
& L_{4}(\varphi)=\varphi^{\prime \prime}-\varphi .
\end{aligned}
$$

with the following properties

$L_{1}\left(C_{1}+C_{2} e^{\xi}+C_{3} e^{-\xi}\right)=0$,

$L_{2}\left(C_{4}+C_{5} e^{\xi}+C_{6} e^{-\xi}\right)=0$,

$L_{3}\left(C_{7} e^{\xi}+C_{8} e^{-\xi}\right)=0$,

$L_{4}\left(C_{9} e^{\xi}+C_{10} e^{-\xi}\right)=0$,

where $C_{i}(i=1$ to 10$)$ are the arbitrary constants.

We construct the zeroth-order deformation equations

$$
\begin{aligned}
& (1-p) L_{1}\left(f(\xi ; p)-f_{0}(\xi)\right)=p \hbar_{1} N_{1}[f(\xi ; p), g(\xi ; p)], \\
& (1-p) L_{2}\left(g(\xi ; p)-g_{0}(\xi)\right)=p \hbar_{2} N_{2}[f(\xi ; p), g(\xi ; p)], \\
& (1-p) L_{3}\left(\theta(\xi ; p)-\theta_{0}(\xi)\right)=p \hbar_{3} N_{3}\left[\begin{array}{l}
f(\xi ; p), g(\xi ; p), \\
\theta(\xi ; p), \varphi(\xi ; p)
\end{array}\right], \\
& (1-p) L_{4}\left(\varphi(\xi ; p)-\varphi_{0}(\xi)\right)=p \hbar_{4} N_{4}\left[\begin{array}{l}
f(\xi ; p), g(\xi ; p), \\
\theta(\xi ; p), \varphi(\xi ; p)
\end{array}\right],
\end{aligned}
$$

subject to the boundary conditions

$$
\begin{aligned}
& f(0 ; p)=0, \quad f^{\prime}(0 ; p)=1, \quad f^{\prime}(\infty ; p)=0, \\
& g(0 ; p)=0, \quad g^{\prime}(0 ; p)=\delta, \quad g^{\prime}(\infty ; p)=0, \\
& \theta^{\prime}(0 ; p)=-B_{i}[1-\theta(0 ; p)], \quad \theta(\infty ; p)=0, \\
& \varphi(0 ; p)=1, \quad \varphi(\infty ; p)=0, \\
& N_{1}[f(\xi ; p), g(\xi ; p)]=\left(1+\frac{1}{\beta}\right) \frac{\partial^{3} f(\xi ; p)}{\partial \xi^{3}} \\
& +(f(\xi ; p)+g(\xi ; p)) \frac{\partial^{2} f(\xi ; p)}{\partial \xi^{2}} \\
& -2\left(\frac{\partial f(\xi ; p)}{\partial \xi}+\frac{\partial g(\xi ; p)}{\partial \xi}\right) \frac{\partial f(\xi ; p)}{\partial \xi}-(M+K) \frac{\partial f(\xi ; p)}{\partial \xi}, \\
& N_{2}[f(\xi ; p), g(\xi ; p)]=\left(1+\frac{1}{\beta}\right) \frac{\partial^{3} g(\xi ; p)}{\partial \xi^{3}} \\
& +(f(\xi ; p)+g(\xi ; p)) \frac{\partial^{2} g(\xi ; p)}{\partial \xi^{2}} \\
& -2\left(\frac{\partial f(\xi ; p)}{\partial \xi}+\frac{\partial g(\xi ; p)}{\partial \xi}\right) \frac{\partial g(\xi ; p)}{\partial \xi}-(M+K) \frac{\partial g(\xi ; p)}{\partial \xi}, \\
& N_{3}[f(\xi ; p), g(\xi ; p) \theta(\xi ; p), \varphi(\xi ; p)]=\left(1+\frac{4}{3} R\right) \frac{\partial^{2} \theta(\xi ; p)}{\partial \xi^{2}} \\
& +\operatorname{Pr}\left((f(\xi ; p)+g(\xi ; p)) \frac{\partial \theta(\xi ; p)}{\partial \xi}\right) \\
& +\operatorname{Pr}\left(\begin{array}{l}
\left.N b \frac{\partial \theta(\xi ; p)}{\partial \xi} \frac{\partial \varphi(\xi ; p)}{\partial \xi}+N t\left(\frac{\partial \theta(\xi ; p)}{\partial \xi}\right)^{2}\right), \\
+Q \theta(\xi ; p)
\end{array}\right)
\end{aligned}
$$


$N_{3}[f(\xi ; p), g(\xi ; p), \theta(\xi ; p), \varphi(\xi ; p)]=\frac{\partial^{2} \varphi(\xi ; p)}{\partial \xi^{2}}$

$+S c(f(\xi ; p)+g(\xi ; p)) \frac{\partial \varphi(\xi ; p)}{\partial \xi}+\left(\frac{N t}{N b}\right) \frac{\partial^{2} \theta(\xi ; p)}{\partial \xi^{2}}$,

When $p=0$ and $p=1$, we obtain

$$
\begin{aligned}
& f(\xi ; 0)=f_{0}(\xi) \quad f(\xi ; 1)=f(\xi), \\
& g(\xi ; 0)=g_{0}(\xi) \quad g(\xi ; 1)=g(\xi), \\
& \theta(\xi ; 0)=\theta_{0}(\xi) \quad \theta(\xi ; 1)=\theta(\xi), \\
& \varphi(\xi ; 0)=\varphi_{0}(\xi) \quad \varphi(\xi ; 1)=\varphi(\xi) \text {. }
\end{aligned}
$$

Thus, as $p$ increases from 0 to 1 then $f(\xi ; \mathrm{p}), g(\xi ; \mathrm{p}), \theta(\xi ; \mathrm{p})$ and $\varphi(\xi ; p)$ vary from initial approximations to the exact solutions of the original nonlinear differential equations.

Now, expanding $f(\xi ; p), g(\xi ; p), \theta(\xi ; p)$ and $\varphi(\xi ; p)$ in Taylor's series w.r.to $p$, we have

$$
f(\xi ; p)=f_{0}(\xi)+\sum_{m=1}^{\infty} f_{m}(\xi) p^{m},
$$

$$
g(\xi ; p)=g_{0}(\xi)+\sum_{m=1}^{\infty} g_{m}(\xi) p^{m},
$$

$$
\theta(\xi ; p)=\theta_{0}(\xi)+\sum_{m=1}^{\infty} \theta_{m}(\xi) p^{m}
$$

$\varphi(\xi ; p)=\varphi_{0}(\xi)+\sum_{m=1}^{\infty} \varphi_{m}(\xi) p^{m}$,

where

$$
\begin{aligned}
& f_{m}(\xi)=\left.\frac{1}{m !} \frac{\partial^{m} f(\xi ; p)}{\partial p^{m}}\right|_{p=0}, \\
& g_{m}(\xi)=\left.\frac{1}{m !} \frac{\partial^{m} g(\xi ; p)}{\partial p^{m}}\right|_{p=0}, \\
& \theta_{m}(\xi)=\left.\frac{1}{m !} \frac{\partial^{m} \theta(\xi ; p)}{\partial p^{m}}\right|_{p=0}, \\
& \varphi_{m}(\xi)=\left.\frac{1}{m !} \frac{\partial^{m} \varphi(\xi ; p)}{\partial p^{m}}\right|_{p=0} .
\end{aligned}
$$

If the initial approximations, auxiliary linear operators and non-zero auxiliary parameters are chosen in such a way that the series (25) to (28) are convergent at $p=1$, then

$$
\begin{aligned}
& f(\eta)=f_{0}(\xi)+\sum_{m=1}^{\infty} f_{m}(\xi), \\
& g(\eta)=g_{0}(\xi)+\sum_{m=1}^{\infty} g_{m}(\xi),
\end{aligned}
$$

$$
\begin{aligned}
& \theta(\xi)=\theta_{0}(\xi)+\sum_{m=1}^{\infty} \theta_{m}(\xi), \\
& \varphi(\xi)=\varphi_{0}(\xi)+\sum_{m=1}^{\infty} \varphi_{m}(\xi) .
\end{aligned}
$$

Differentiating Eqs. (15) to (19) $m$ times w.r.to $p$, setting $p=0$ and finally dividing with $m$ !, we get the mth-order deformation equations as follows:

$$
\begin{aligned}
& L_{1}\left(f_{m}(\xi)-\chi_{m} f_{m-1}(\xi)\right)=\hbar_{1} R_{m}^{f}(\xi), \\
& L_{2}\left(g_{m}(\xi)-\chi_{m} g_{m-1}(\xi)\right)=\hbar_{2} R_{m}^{g}(\xi), \\
& L_{3}\left(\theta_{m}(\xi)-\chi_{m} \theta_{m-1}(\xi)\right)=\hbar_{3} R_{m}^{\theta}(\xi), \\
& L_{4}\left(\varphi_{m}(\xi)-\chi_{m} \varphi_{m-1}(\xi)\right)=\hbar_{4} R_{m}^{\varphi}(\xi),
\end{aligned}
$$

with the following boundary conditions

$$
\begin{array}{lrr}
f_{m}(0)=0, & f_{m}^{\prime}(0)=0, & f_{m}^{\prime}(\infty)=0, \\
g_{m}(0)=0, & g_{m}^{\prime}(0)=0, & g_{m}^{\prime}(\infty)=0, \\
\theta_{m}^{\prime}(0)=0, & \theta_{m}(\infty)=0, \\
\varphi_{m}(0)=0, & \varphi_{m}(\infty)=0,
\end{array}
$$

where

$$
\begin{aligned}
& R_{m}^{f}(\xi)=\left(1+\frac{1}{\beta}\right) f_{m-1}^{\prime \prime \prime}+\sum_{i=0}^{m-1}\left(f_{m-1-i}+g_{m-1-i}\right) f_{i}^{\prime \prime} \\
& -2 * \sum_{i=0}^{m-1}\left(f_{m-1-i}^{\prime}+g_{m-1-i}^{\prime}\right) f_{i}^{\prime}-(M+K) f_{m-1}^{\prime}, \\
& R_{m}^{g}(\xi)=\left(1+\frac{1}{\beta}\right) g_{m-1}^{\prime \prime \prime}+\sum_{i=0}^{m-1}\left(f_{m-1-i}+g_{m-1-i}\right) g_{i}^{\prime \prime} \\
& -2 * \sum_{i=0}^{m-1}\left(f_{m-1-i}^{\prime}+g_{m-1-i}^{\prime}\right) g_{i}^{\prime}-(M+K) g_{m-1}^{\prime}, \\
& R_{m}^{\theta}(\xi)=\left(1+\frac{4 R}{3}\right) \theta_{m-1}^{\prime \prime}+\operatorname{Pr}\left(\begin{array}{l}
\sum_{i=0}^{m-1}\left(f_{m-1-i}+g_{m-1-i}\right) \theta_{i}^{\prime} \\
+N b \sum_{i=0}^{m-1} \theta_{m-1-i}^{\prime} \varphi_{i}^{\prime} \\
+N \sum_{i=0}^{m-1} \theta_{m-1-i}^{\prime} \theta_{i}^{\prime} \\
+Q \theta_{m-1}^{\prime}
\end{array}\right),
\end{aligned}
$$

$$
\begin{aligned}
& R_{m}^{\varphi}(\xi)=\varphi_{m-1}^{\prime \prime}+S c\left(\sum_{i=0}^{m-1}\left(f_{m-1-i}+g_{m-1-i}\right) \varphi_{i}^{\prime}\right) \\
& +\left(\frac{N t}{N b}\right) \theta_{m-1}^{\prime \prime}, \\
& \chi_{m}= \begin{cases}0, & m \leq 1, \\
1, & m>1 .\end{cases}
\end{aligned}
$$


If we let $f_{m}^{*}(\xi), g_{m}^{*}(\xi), \theta_{m}^{*}(\xi)$ and $\varphi_{m}^{*}(\xi)$ as the special solutions of mth order deformation equations, then the general solution is given by

$$
\begin{aligned}
f_{m}(\xi) & =f_{m}^{*}(\xi)+C_{1}+C_{2} e^{\xi}+C_{3} e^{-\xi}, \\
g_{m}(\xi) & =g_{m}^{*}(\xi)+C_{4}+C_{5} e^{\xi}+C_{6} e^{-\xi}, \\
\theta_{m}(\xi) & =\theta_{m}^{*}(\xi)+C_{7} e^{\xi}+C_{8} e^{-\xi}, \\
\varphi_{m}(\xi) & =\varphi_{m}^{*}(\xi)+C_{9} e^{\xi}+C_{10} e^{-\xi},
\end{aligned}
$$

where the integral constants $C_{i}(i=1$ to 10$)$ are determined using the boundary conditions.

\section{CONVERGENCE OF HAM}

The convergence and rate of approximation of the procured results strongly rely on on the auxiliary parameters $\hbar_{1}, \hbar_{2}, \hbar_{3}$ and $\hbar_{4}$. To acquire the relevant values for these parameters, $\hbar$-curves are portrayed in Figure 2. From this diagrammatic representation, it is scrutinized that the plausible region of the parameters is about $[-0.94,0.0]$. For $\hbar_{1}=\hbar_{2}=\hbar_{3}=\hbar_{4}=-0.52$, the series solutions are convergent in the whole region of $\xi$. Table 1 displays the convergence of the method.

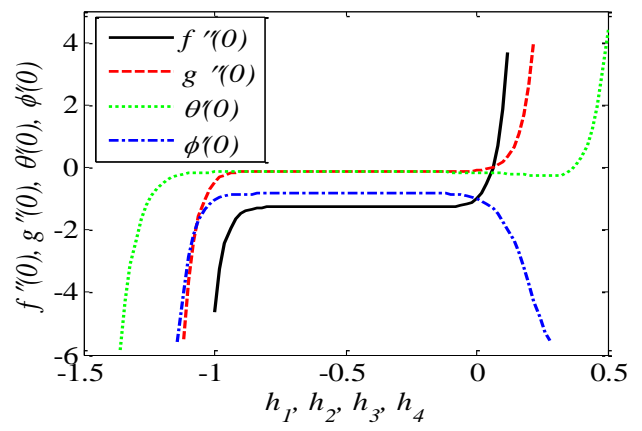

Figure 2. $\hbar$-curves for $f^{\prime \prime}(0), g^{\prime \prime}(0), \theta^{\prime}(0)$ and $\varphi^{\prime}(0)$ at $15^{\text {th }}$ order approximations

Table 1. Convergence of HAM solution for different orders of approximations when

\begin{tabular}{|r|c|c|c|c|}
\hline Order & $-f^{\prime \prime}(0)$ & $-g "(0)$ & $-\theta^{\prime}(0)$ & $-\varphi^{\prime \prime}(0)$ \\
\hline 5 & 1.903651 & 0.190365 & 0.169773 & 0.834825 \\
\hline 10 & 1.903677 & 0.190368 & 0.167740 & 0.844751 \\
\hline 15 & 1.903677 & 0.190368 & 0.167613 & 0.844049 \\
\hline 20 & 1.903677 & 0.190368 & 0.167591 & 0.844287 \\
\hline 25 & 1.903677 & 0.190368 & 0.167588 & 0.844253 \\
\hline 30 & 1.903677 & 0.190368 & 0.167588 & 0.844256 \\
\hline 35 & 1.903677 & 0.190368 & 0.167588 & 0.844256 \\
\hline 40 & 1.903677 & 0.190368 & 0.167588 & 0.844256 \\
\hline
\end{tabular}

\section{RESULTS AND DISCUSSION}

In this, we panoply some results which bring acumen about the problem. For these computations, we consider the following values all over the study except refurbished values as delivered in the tables and graphs. The results are taken for Newtonian and Casson fluids.

$$
\begin{aligned}
& M=0.5, \delta=K=R=0.1, \operatorname{Pr}=2.0, N b=N t=0.2, \\
& Q=0.1, B_{i}=0.2, S c=2.0 .
\end{aligned}
$$

Lorentz force impinges its resistance on fluid motion as a result axial and transverse velocities get decelerated with Hartmann parameter $M$. This is shown in Figures 3 and 4. Figures 5 and 6 depict that the velocities in both directions diminish as the porous parameter $K$ enhances. This is due to the Darcy resistance provided by the porous medium. Figures 7 and 8 illustrate the impact of ratio parameter $\delta$ on both the velocities. Amplification in $\delta$ implies an immensive stretching rate in the $y$-direction and thus the velocity in the $y$-direction raises whereas the velocity in the $x$-direction decelerates correspondingly. An increase in Prandtl number Pr constitutes devaluation in the temperature field. Physically larger Prandtl number has feeble thermal diffusivity due to which lower temperature is observed in Figure 9. It is noticed from Figure 10 that a raise in radiation parameter $R$ has the capability to enhance the thermal boundary layer and consequently the fluid temperature rises.

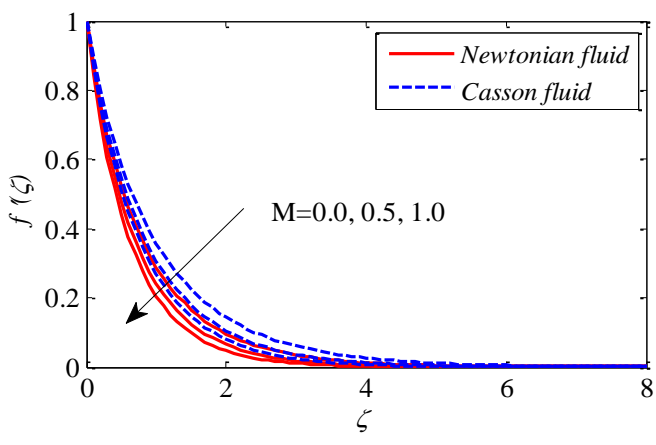

Figure 3. Effect of $M$ on $f^{\prime}(\xi)$

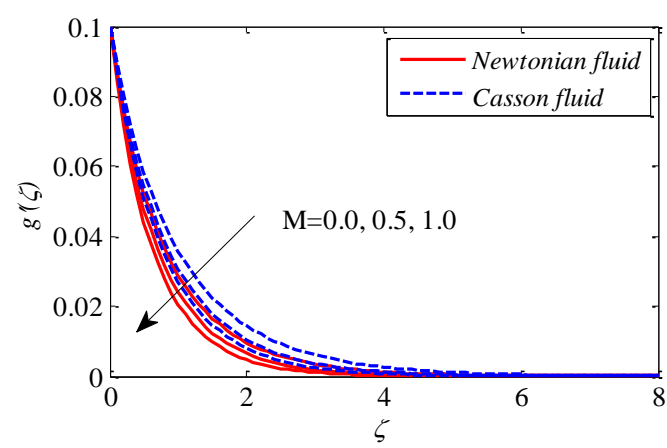

Figure 4. Effect of $M$ on $g^{\prime}(\xi)$

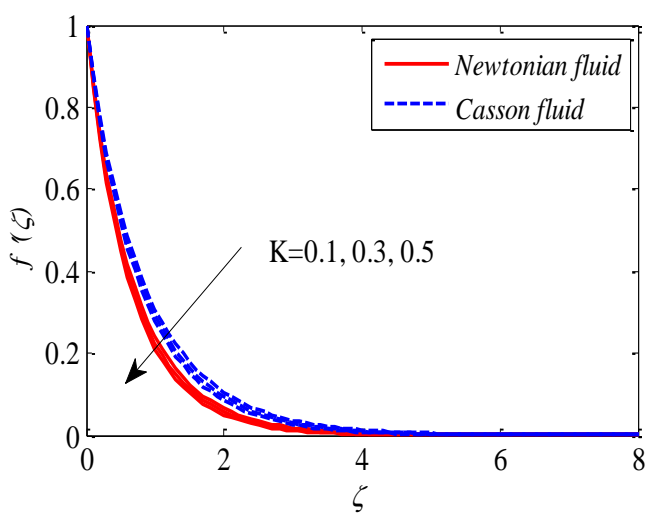

Figure 5. Effect of $K$ on $f^{\prime}(\xi)$ 


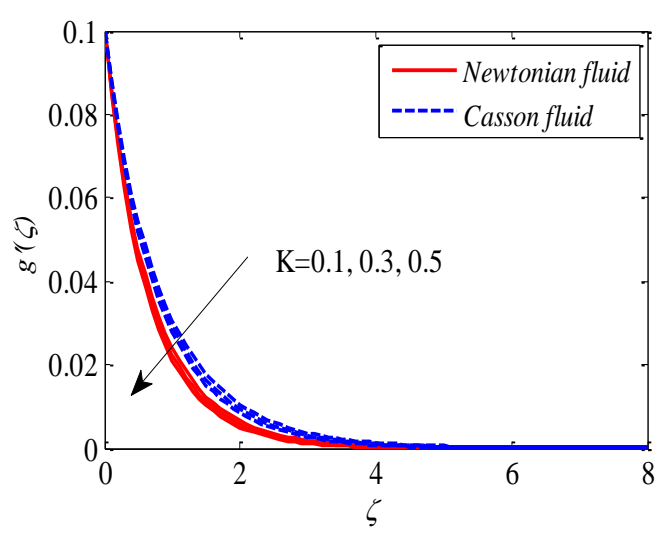

Figure 6. Effect of $K$ on $g^{\prime}(\xi)$

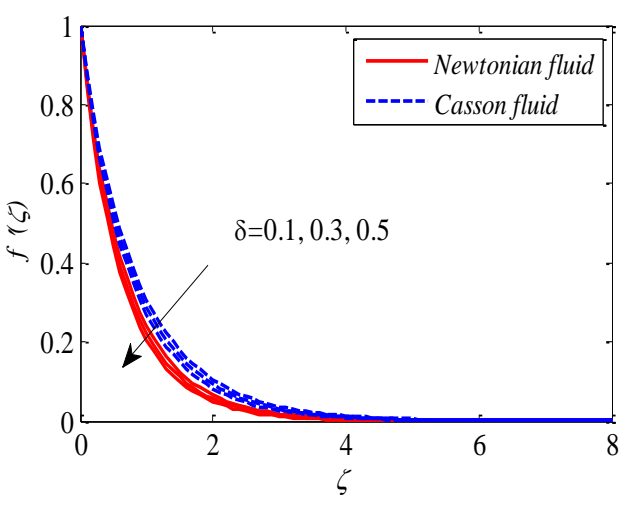

Figure 7. Effect of $\delta$ on $f^{\prime}(\xi)$

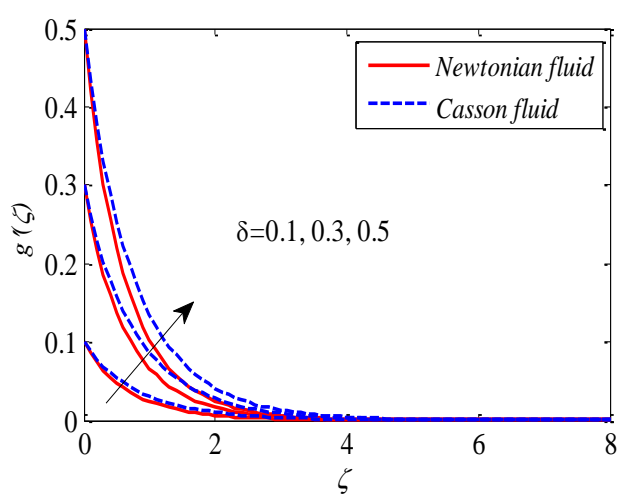

Figure 8. Effect of $\delta$ on $g^{\prime}(\xi)$

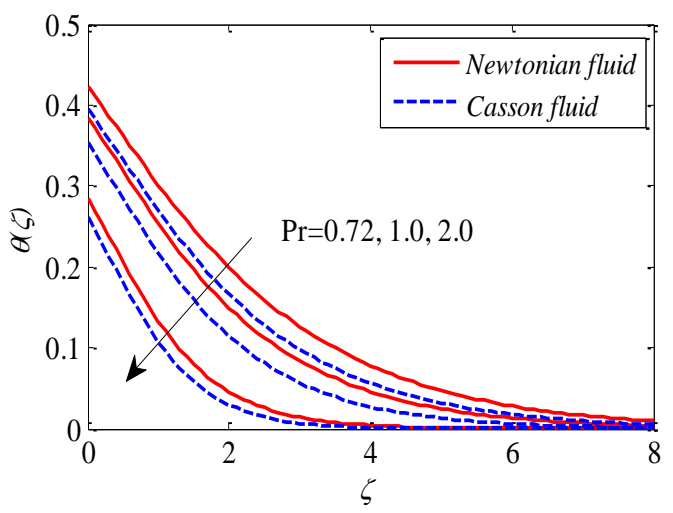

Figure 9. Effect of $\operatorname{Pr}$ on $\theta(\xi)$

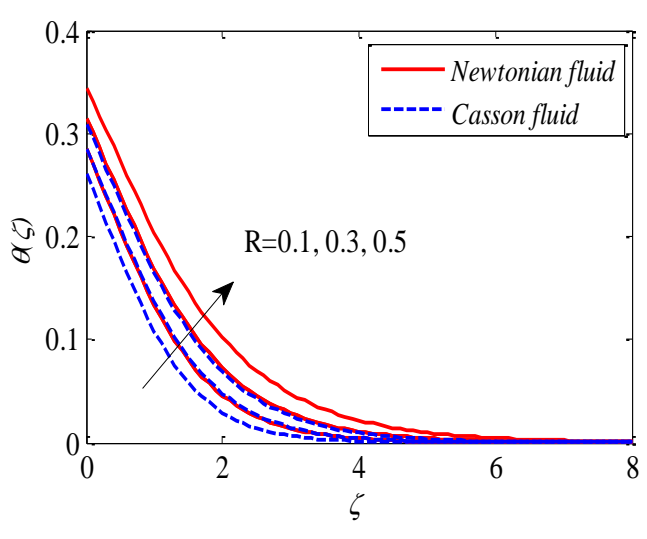

Figure 10. Effect of $R$ on $\theta(\xi)$

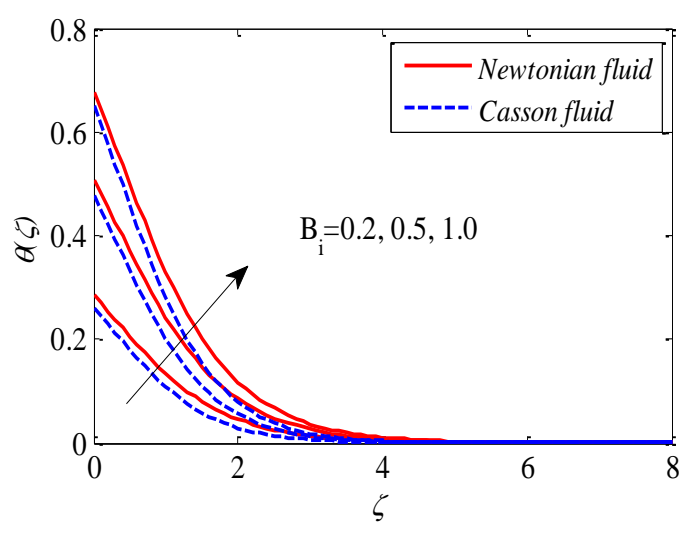

Figure 11. Effect of $B_{i}$ on $\theta(\xi)$

Figure 11 illustrate that enhancing values of Biot number $B_{i}$ uplift the temperature. Physically an increase in Biot number causes an enhancement in the heat transfer coefficient which up lift the thermal energy to the fluid and thus temperature increases. In the existence of heat source parameter $Q$ in the fluid, thermal energy is released and as a repercussion, temperature increases. This is shown in Figure 12. The influence of Brownian motion and thermophoresis parameters $N b$ and $N t$ on temperature field is given in Figurs 13 and 14. These parameters are arrived due to the existence of nanoparticles which enhance the thermal conductivity of the fluid as a result temperature increases with $N b$ and $N t$. It is observed that the impact of these parameters is significantly high in Newtonian fluid while compared with the Casson fluid. The effect of Brownian motion and thermophoresis parameters $N b$ and $N t$ on concentration field is given in Figurs 15 and 16. Enhancing values of $N t$ shows stronger thermophoretic force which deviates the nanoparticles from the hot sheet to the quiescent fluid thereby increasing the nanoparticle volume fraction boundary layer. Hence concentration enhances with $N t$. But we pointed out an opposite phenomena in the concentration profiles with an increase in the Brownian motion parameter. Figure 17 indicates the influence of Schmidt number $S c$ on concentration profiles. It is observed that concentration depreciates with $S c$. It is due to the fact that increasing values of $S c$ results a weaker Brownian coefficient. From the Figurs 18 and 19, it is observed that the skin friction coefficients are decelerating with the increasing values of magnetic parameter $M$ and porous parameter $K$. Local Nusselt number increases with Biot number $B_{i}$ and 
radiation parameter $R$. This is shown in Figure 20. Sherwood number is enhancing with the increase of Schmidt number $S c$ and diminishing with thermophoresis parameter $N t$. This is given in Figure. 21.

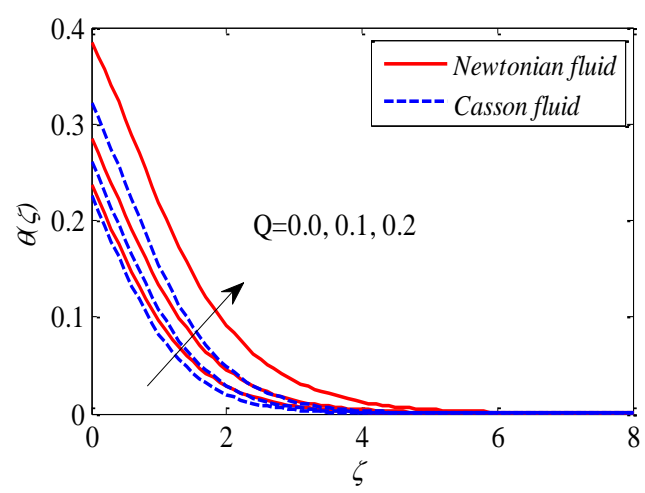

Figure 12. Effect of $Q$ on $\theta(\xi)$

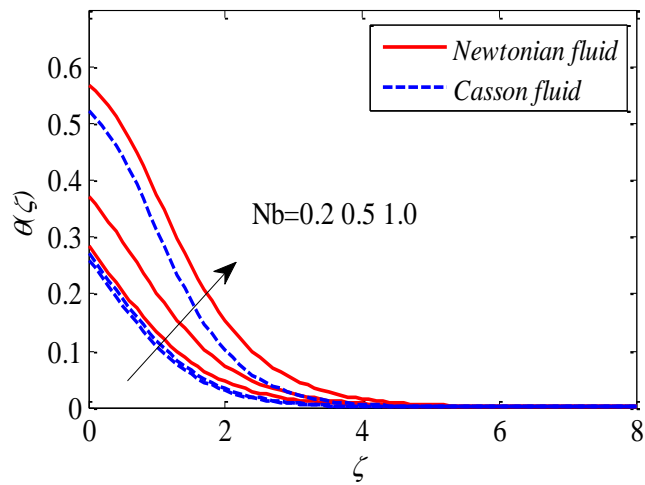

Figure 13. Effect of $N b$ on $\theta(\xi)$

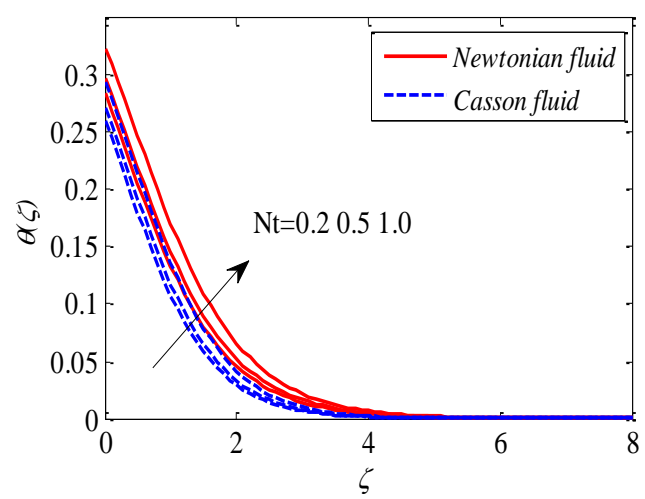

Figure 14. Effect of $N t$ on $\theta(\xi)$

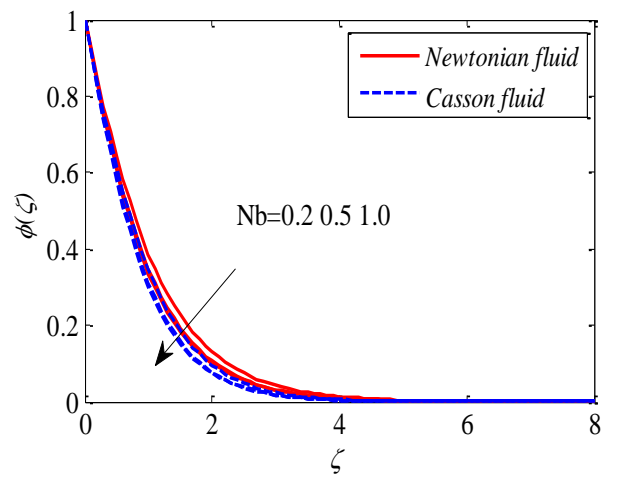

Figure 15. Effect of $N b$ on $\varphi(\xi)$

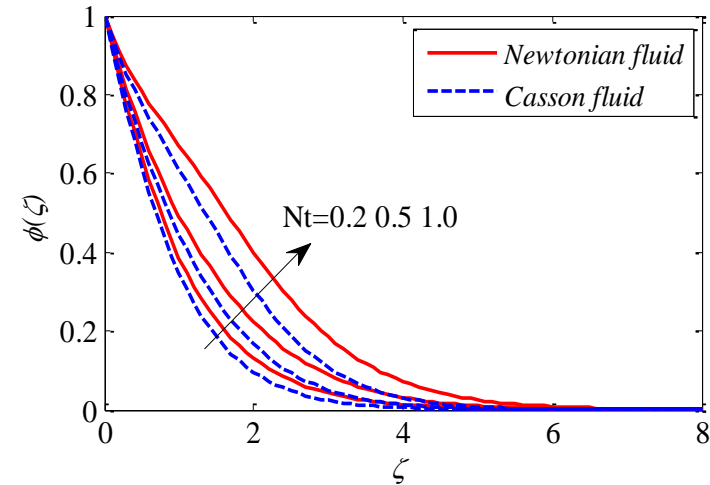

Figure 16. Effect of $N t$ on $\varphi(\xi)$

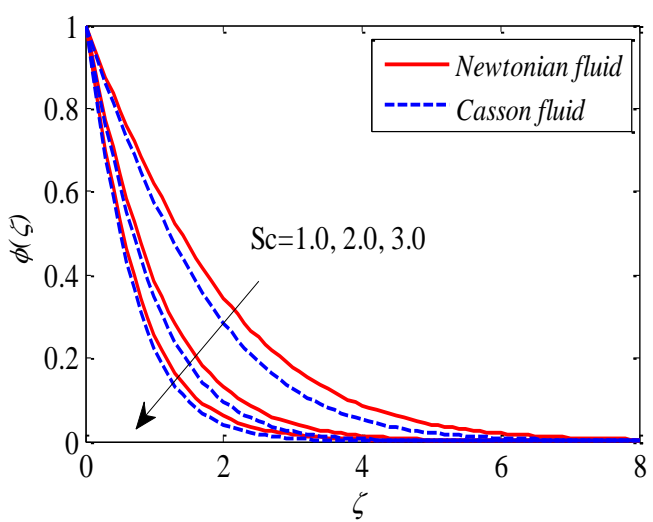

Figure 17. Effect of $S c$ on $\varphi(\xi)$

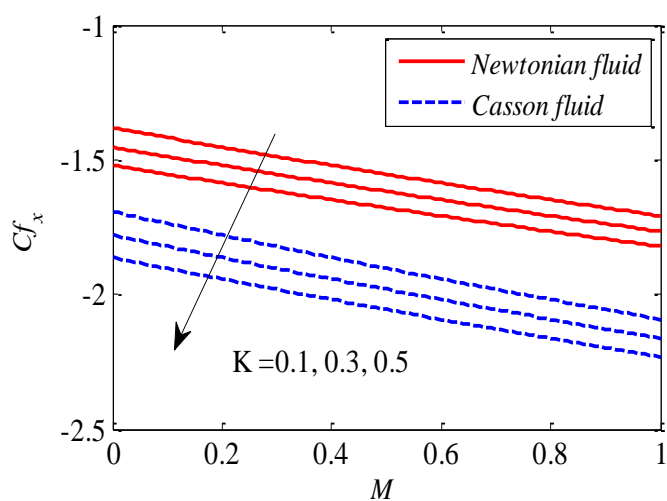

Figure 18. Effect of $M$ and $K$ on $C_{f x}$

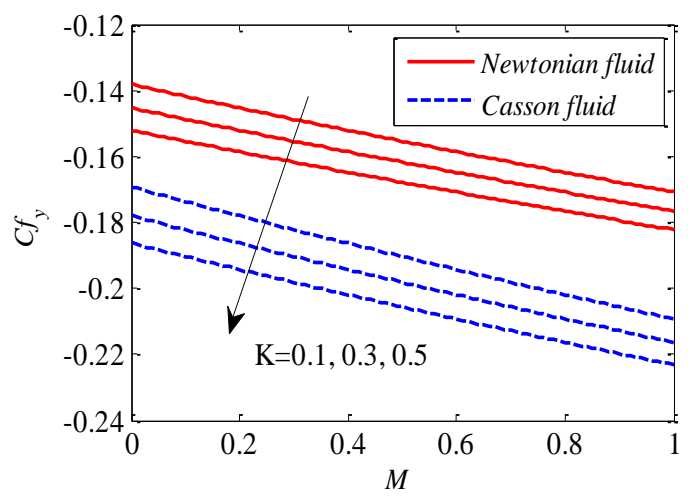

Figure 19. Effect of $M$ and $K$ on $C_{f y}$ 


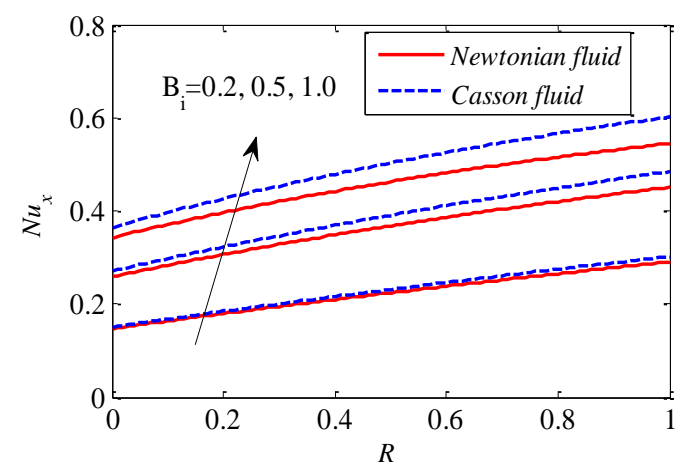

Figure 20. Effect of $B_{i}$ and $R$ on $N u_{x}$

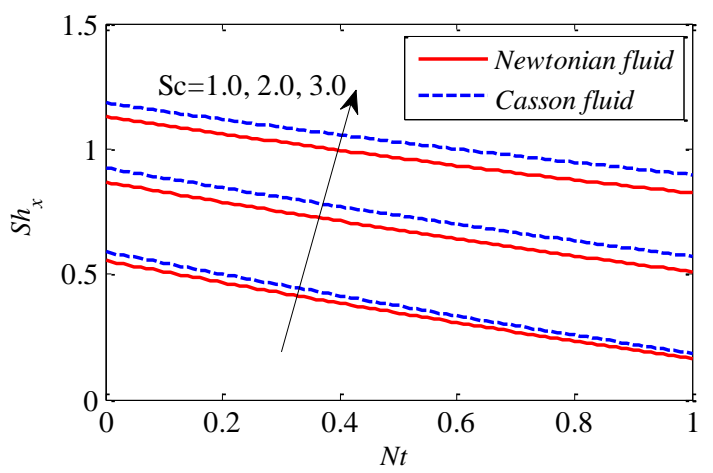

Figure 21. Effect of $N t$ and $S c$ on $S h_{x}$

To endorse the veracity of the present analysis, the obtained results of $-f^{\prime \prime}(0)$ and have been compared with that of Liu et al. [12] solutions for the limiting case and.

Table 2. Comparison of and

\begin{tabular}{|c|c|c|c|c|}
\hline \multirow{2}{*}{} & \multicolumn{2}{|c|}{ Liu et al. [12] } & \multicolumn{2}{c|}{ HAM } \\
\cline { 2 - 5 } & & & & 0 \\
\hline 0.0 & 1.28180856 & 0 & 1.281826 & 0 \\
\hline 0.5 & 1.56988846 & 0.78494423 & 1.569889 & 0.784944 \\
\hline 1.0 & 1.81275105 & 1.81275105 & 1.812752 & 1.812752 \\
\hline
\end{tabular}

\section{CONCLUSIONS}

In this article a detailed scrutiny is accomplished to study the three dimensional steady incompressible MHD flow of Casson nanofluid over an exponentially stretching sheet with convective boundary condition under radiation and heat source. Brownian motion and thermophoresis effects are considered in the flow region. HAM is used to solve the nonlinear ordinary differential equations. Following are the major findings of the analysis:

- Increase in reduces the axial velocity where as reverse trend is observed in transverse velocity.

- The impact of and is significantly high in Newtonian fluid while compared with the Casson fluid.

- Schmidt number has propensity to decelerate the concentration field.

- It is seen that an increase in radiation parameter causes more impact on temperature field of the Newtonian fluid when compared with the Casson fluid.

\section{REFERENCES}

[1] Choi SUS. (1995). Enhancing thermal conductivity of fluids with nanoparticles. Proceedings of the 1995 ASME International Mechanical Engineering Congress and Exposition, San Francisco, 99-105.

[2] Prabhat N, Buongiorno J, Hu LW. (2012). Convective heat transfer enhancement in nanofluids: Real anomaly or analysis artifact. Journal of Nanofluids 1(1): 55-62. https://doi.org/ 10.1166/jon.2012.1003

[3] Khan WA, Pop I. (2010). Boundary layer flow of a nanofluid past a stretching sheet. Int. J. Heat Mass Transfer 53(11-12): 2477-2483. https://doi.org/ 10.1016/j.ijheatmasstransfer.2010.01.032

[4] Hady FM, Ibrahim FS, Abdel-Gaied SM, Eid MR. (2012). Radiation effect on viscous flow of a nanofluid and heat transfer over a nonlinearly stretching sheet. Nanoscale Research Letters 7: 229-242. https://doi.org/ 10.1186/1556-276X-7-229

[5] Rida SZ, Mohamed RA, Mubarak MS. (2016). MHD nanofluid flow over a stretching sheet through a porous medium with heat generation and thermal radiation. Journal of Nanofluids 5(1): 130-138. https://doi.org/ 10.1166/jon.2016.1187

[6] Mabood F, Khan WA, Ismail AIM. (2015). MHD boundary layer flow and heat transfer of nanofluids over a nonlinear stretching sheet: A numerical study. J. Magn. Magn. Mater 374: 569-576. https://doi.org/ 10.1016/j.jmmm.2014.09.013

[7] Haq RU, Nadeem S, Khan ZH, Sher Akbar N. (2015). Thermal radiation and slip effects on MHD stagnation point flow of nanofluid over a stretching sheet. Physica E: Low-dimensional system and Nanostructure 65: 1723. https://doi.org/ 10.1016/j.physe.2014.07.013

[8] Raashidi MM, Vishnu Ganesh N, Abdul Hskeem AK, Ganga B. (2014). Buoyancy effect on MHD flow of nanofluid over a stretching sheet in the presence of thermal radiation. J. Mol. Liq. 198: 234-238. https://doi.org/ 10.1016/j.molliq.2014.06.037

[9] Nadeem N, Haq RU, Khan ZH. (2014). Numerical solution of non-Newtonian nanofluid over a stretching sheet. Applied Nanoscience 4(5): 625-631. https://doi.org/ 10.1007/s13204-013-0235-8

[10] Wang CY. (1984). The three-dimensional flow due to a stretching flat surface. Phys. Fluids 27: 1915-1917. https://doi.org/ 10.1063/1.864868

[11] Ariel PD. (2007). On computation of the threedimensional flow past a stretching sheet. Appl. Math. Comput. 188(2): 1244-1250. https://doi.org/ 10.1016/j.amc.2006.10.083

[12] Liu IC, Wang HH, Peng YF. (2014). Flow and heat transfer for three dimensional flow over an exponentially stretching surface. Chem. Eng, Commun. 200(2): 253268. https://doi.org/ 10.1080/00986445.012.703148

[13] Hayat T, Shehzad SA, Alsaedi A. (2014). MHD threedimensional flow by an exponentially stretching surface with convective boundary condition. J. Aerosp. Eng. 27(4): $04014011 . \quad$ https://doi.org/ 10.1061/(ASCE)AS.1943-5525.0000360

[14] Nadeem S, Haq RU, Khan ZH. (2014). Heat transfer analysis of water-based nanofluid over an exponentially stretching sheet. Alexandria Engineering Journal 53: 219-224, 2014. https://doi.org/ 10.1016/j.aej.2013.11.003 
[15] Jayachandra babu M, Sandeep N. (2016). Threedimensional MHD slip flow of nanofluids over a slendering stretching sheet with thermophoresis and Brownian motion effects. Advanced Power Technology 27(5): 2039-2050. https://doi.org/ 10.1016/japt.2016.07.013

[16] Ahmed N, Kalita H, Barua D. (2012). An oscillatory three dimensional flow past an infinite vertical porous plate with soret and Dufour effects. International Journal of Heat and Technology 30(2): 9-18. https://doi.org/ 10.18280/ijht.300202

[17] Casson N. (1959). A flow equation for pigment oil suspensions of the printing ink type. Rheology of Disperse Systems: Proceedings of a Conference Organized by the British Society of Rheology., New York, pp. 84-104.

[18] Mukhopadhyay S. (2013). Casson fluid flow and heat transfer over a nonlinear surface. Chinese. Phy. B. 22(7): 074701. https://doi.org/ 10.1088/16741056/22/7/074701

[19] Mustafa M, Khan JA. (2015). Model for flow of Casson nanofluid past a non-linearly stretching sheet considering magnetic effects. AIP Advances 5: 07748. https://doi.org/ 10.1063/1.4927449

[20] Ibrahim W, Makinde OD. (2016). Magnetohydrodynamic stagnation point flow and heat transfer of Casson nanofluid past a stretching sheet with slip and convective boundary conditions. J. Aerosp. Eng. 29(2): $04015037 . \quad$ https://doi.org/ 10.1061/(ASME)AS/1943-5525.0000529

[21] Mustafa M, Mushtaq A, Hayat T, Alsaedi A. (2015). Radiation effects in three-dimensional flow over a bidirectional exponentially stretching sheet. J. Taiwan. Inst. Chem. E. 47: 43-49. https://doi.org/ 10.1016/j.jtice.2014.10.011

[22] Butt AS, Tufail MN, Asif Ali. (2016). Threedimensional flow of a magnetohydrodynamic Casson fluid over an unsteady stretching sheet embedded into a porous medium. J. Appl. Mech. Tech. Phys. 57(2): 283 292. https://doi.org/ 10.1134/S00218944160220115

[23] El-Aziz MA. (2007). Temperature dependent viscosity and thermal conductivity effects on combined heat and mass transfer in MHD three-dimensional flow over a stretching surface with Ohmic heating. Meccanica 42(4): 375-386. https://doi.org/ 10.1007/s11012-0069051-5

[24] Mahanta G, Shaw S. (2015). 3D Casson fluid flow past a porous linearly stretching sheet with convective boundary condition. Alexandria Engineering Journal 54: 653-659. https://doi.org/ 10.1016/j.aej.2015.04.014

[25] Sulochana C, Ashwinkuar GP, Sandeep N. (2016). Similarity solution of 3D Casson nanofluid flow over a stretching sheet with convective boundary conditions. Journal of Nigerian Mathematical Society 35: 128-141. https://doi.org/ 10.1016/j.jnnms.2016.01.001

[26] Liao SJ. (2012). Homotopy analysis method in nonlinear differential equations. Springer \& Higher Education Press, Heidelberg.

[27] Hayat T, Shehzad SA, Alsaedi A. (2012). Soret and Dufour effects on magnetohydrodynamic (MHD) flow of Casson fluid. Appl. Math. Mech. -Engl. Ed. 33(10): 1301-1312. https://doi.org/ 10.1007/s10483-012-16236

[28] Ibrahim SM, Kumar PV, Lorenzini G, Mabood F. (2017). Numerical study of the onset of chemical reaction and heat source on dissipative MHD stagnation point flow of Casson nanofluid over a nonlinear stretching sheet with velocity slip and convective boundary conditions. J. Eng. Thermophys 26(2): 256-271. https://doi.org/ 11.1134/S18102328170200096

[29] Kumar PV, Ibrahim SM, Lorenzini G. (2017). Impact of thermal radiation and Joule heating on MHD mixed convection flow of a Jeffrey fluid over a stretching sheet using homotopy analysis method. International Journal of Heat and Technology 35(4): 978-986. https://doi.org/ 10.18280/ijht.350434

[30] Ibrahim SM, Lorenzini G, Vijaya Kumar P, Raju CSK. (2017). Influence of chemical reaction and heat source on dissipative MHD mixed convection flow of a Casson nanofluid over a nonlinear permeable stretching sheet. Int. J. HeatMass Trans. 111: 346-355. https://doi.org/ 10.1016/j.ijheatmasstransfer.2017.03.097

\section{NOMENCLATURE}

\begin{tabular}{|c|c|}
\hline $\mathrm{u}, \mathrm{v}, \mathrm{w}$ & $\begin{array}{l}\text { velocity components in } x, y, z \text { directions, } \\
\mathrm{m} . \mathrm{s}^{-1}\end{array}$ \\
\hline $\mathrm{B}_{0}$ & $\begin{array}{l}\text { uniform magnetic field strength, N. } \mathrm{m}^{-} \\
{ }^{1} . \mathrm{A}^{-1}\end{array}$ \\
\hline $\mathrm{K}^{*}$ & permeable parameter \\
\hline $\mathrm{T}$ & Temperature, $\mathrm{K}$ \\
\hline $\mathrm{C}$ & nanoparticle concentration \\
\hline $\mathrm{T}_{\mathrm{f}}$ & $\begin{array}{l}\text { convective fluid temperature below the } \\
\text { sheet, } \mathrm{K}\end{array}$ \\
\hline $\mathrm{C}_{\mathrm{w}}$ & $\begin{array}{l}\text { nanoparticle concentration at } \\
\text { the surface of the sheet }\end{array}$ \\
\hline $\mathrm{T}_{\infty}$ & ambient fluid temperature, $\mathrm{K}$ \\
\hline $\mathrm{C}_{\infty}$ & ambient nanoparticle concentration \\
\hline $\mathrm{D}_{\mathrm{B}}$ & Brownian diffusion coefficient, $\mathrm{m}^{2} . \mathrm{s}^{-1}$ \\
\hline $\mathrm{D}_{\mathrm{T}}$ & $\begin{array}{l}\text { thermophoretic diffusion coefficient, } \\
\mathrm{m}^{2} . \mathrm{s}^{-1}\end{array}$ \\
\hline $\mathrm{U}_{\mathrm{w}}, \mathrm{V}_{\mathrm{w}}$ & stretching velocities, $\mathrm{m} . \mathrm{s}^{-1}$ \\
\hline $\mathrm{U}_{0}, \mathrm{~V}_{0}$ & reference velocities, $\mathrm{m} . \mathrm{s}^{-1}$ \\
\hline $\mathrm{L}$ & reference length \\
\hline M & Hartman number \\
\hline $\mathrm{Q}_{0}$ & heat source coefficient \\
\hline $\operatorname{Pr}$ & Prandtl number \\
\hline $\mathrm{Nb}$ & Brownian motion parameter \\
\hline $\mathrm{R}$ & radiation parameter \\
\hline $\mathrm{Nt}$ & thermophoresis parameter \\
\hline $\mathrm{K}$ & non-dimensional porous parameter \\
\hline Q & heat source parameter \\
\hline $\mathrm{Sc}$ & Schmidt number \\
\hline $\operatorname{Re}$ & local Reynolds number \\
\hline $\mathrm{q}_{\mathrm{r}}$ & radiative heat flux, W. $\mathrm{m}^{-1}$ \\
\hline $\mathrm{q}_{\mathrm{w}}$ & wall heat flux \\
\hline $\mathrm{q}_{\mathrm{m}}$ & wall mass flux \\
\hline $\mathrm{C}_{\mathrm{fx}}, \mathrm{C}_{\mathrm{fy}}$ & $\begin{array}{l}\text { skin friction coefficients along the } x \text { and } \\
y \text { directions }\end{array}$ \\
\hline $\mathrm{Nu}_{x}$ & local Nusselt number \\
\hline $\mathrm{Sh}_{\mathrm{x}}$ & Sherwood number \\
\hline
\end{tabular}


$v_{\mathrm{f}}$

$\sigma$

$\rho_{\mathrm{f}}$

$\alpha_{\mathrm{f}}$

$\tau$

$\beta$

$\mathrm{h}_{\mathrm{f}}$

$\mathrm{k}_{\mathrm{f}}$ kinematic viscosity, $\mathrm{m}^{2}$. $\mathrm{s}^{-1}$

electrical conductivity of the base fluid, s. $\mathrm{m}^{-1}$

fluid density, $\mathrm{kg} . \mathrm{m}^{-3}$

thermal diffusivity, $\mathrm{m}^{2} . \mathrm{s}^{-1}$

ratio between effective heat capacity of nanoparticle material and heat capacity of the fluid

Casson fluid parameter

convective heat transfer

coefficient of the fluid

thermal conductivity of the fluid, W. $\mathrm{m}^{-1}$. $\mathrm{k}^{-1}$
$\mathrm{B}_{\mathrm{i}}$

$\mu_{\mathrm{f}}$

$\tau_{\mathrm{wx}}, \tau_{\mathrm{wy}}$

$f^{\prime}, g^{\prime}$

$\theta$

$\varphi$

$\mathrm{k}^{*}$

$\xi$
Stefan-Boltzman constant

ratio parameter

Biot number

dynamic viscosity of the fluid, Pa.s $\mathrm{s}^{-1}$

shear stresses along the $x$ and $y$

directions

velocity profiles

temperature profile

concentration profile

mean absorption coefficient

similarity variable 